\title{
ЛИТЕРАТУРНЫЕ ОБЗОРЫ
}

УДК: 618.1-089; 617-089.844

\section{СОВРЕМЕННЫЕ МЕТОДЫ ЛЕЧЕНИЯ НЕДЕРЖАНИЯ МОЧИ: ПЕТЛЕВЫЕ ОПЕРАЦИИ И МИНИСЛИНГИ (ЧАСТЬ 1)}

\author{
Куприянов Ю.А., Гвоздев М.Ю., Касян Г.Р., Пушкарь Д.Ю. \\ Кафедра урологии, ГБОУ ВПО Московский государственный медико- \\ стоматологический университет Минздравсоцразвития России, г.Москва \\ Адрес: 125206, г.Москва, ул. Вучетича, 21, корпус 3, тел. (495)6113129 \\ Эл.почта: dr.kupriyanov@mail.ru, gvozdev@mail.ru, g.kasyan@gmail.com, pushkardm@mail.ru
}

\begin{abstract}
В течение последнего столетия различные петлевые операџии используются при лечении недержания мочи при напряжении. Методы лечения недержания мочи неразрывно связаны с развитием урологши как науки. Доминирование концепции о сфинктерной недостаточности как основной причины недержания мочи привело киспользованию различных мылечных петель, которые, как полагали многие авторы, могли нести функциональную нагрузку. Со временем различные петли стали применяться для создания дозированной инфравезикальной обструкции в области шейки мочевого пузыря. Последнее десятилетие было ознаменовано значительными успехами и достижениями в лечении недержания мочи у женщин. Полученные сегодня данные дают право говорить, что, в целом, применение синтетических петель, устанавливаемых без натяжения в области средней уретры, позволяет избавить от недержания мочи значительное количество больных с этой медико-сочиальной проблемой во всем мире. Можно сказать, что с появлением минислингов и их использовании, в лечении недержсания мочи появился новый этап. Конщепџия использования минислингов, для имплантаџии которых хирургу требуется один разрез под средней третью уретры, не является новаторской. Так Sтіth представил свою минипетлю, выполненную из кожи свиньи в 1987 году. Первым доступным для использования минислингом стал минислинг TVТ Secur компании Gynаесаге (Ethicon) в 2005 году. На сегодняшний день существует множество минипеmель: TVT Secur $\AA$, MiniArc ${ }^{\circledR}$, Ophira ${ }^{\circledR}$, Adjust $\AA$, Solyx $\AA$, Zipper Sling ${ }^{\circledR}$, Prosurg $\AA$, Tissue Fixation System $\AA$, Needleless $\AA$, Altis $\AA$, Just-swing $\AA$, Desara $\AA$. Многие производители не останавливаются и продолжсют разрабатывать новые минислинги с новыли механизмами фиксации.
\end{abstract}

Ключевые слова: минислинги, недержание мочи, минипетли, синтетические петли

\section{MODERN METHODS FOR TREATING URINARY INCONTINENCE: LOOP OPERATIONS AND MINI-SLINGS (PART ONE)}

\author{
Kupriyanov Y.A., Gvozdev M.Y., Kasyan G.R., Pushkar D.Y.
}

Department of Urology Moscow State University Of Medicine And Dentistry, Moscow

During the last century various loop operations are used in the treatment of stress urinary incontinence. Methods for treating urinary incontinence are inextricably linked with the development of urology as a science. Dominance concept of sphincter deficiency as the main cause of urinary incontinence led to the use of various muscle loops, which was considered by many authors, could carry the functional load. The last decade was marked by significant developments and achievements in the treatment of urinary incontinence in women. Data received today entitle say that, in general, the use of synthetic loops installed without tension in the middle of the urethra, to get rid of urinary incontinence significant number of patients with this medical and social problem worldwide. We can say that with the advent of SIMS and their use in the treatment of urinary incontinence, a new stage. The concept of using SIMS for implantation which the surgeon requires one incision under the middle third of the urethra, is not innovative. So Smith presented his minisling made of pig skin in 1987. First available for use SIMS became minisling TVT Secur company Gynaecare (Ethicon) in 2005. To date, there are many SIMS: TVT Secur $\AA$, MiniArc ${ }^{\circledR}$, Ophira ${ }^{\circledR}$, Adjust $\AA$, Solyx $\AA$, Zipper Sling $\AA$, Prosurg ${ }^{\circledR}$, Tissue Fixation System $\AA$, Needleless ${ }^{\circledR}$, Altis $\AA$, Just-swing $\AA$, Desara $\AA$. Many manufacturers do not stop and continue to develop new minislingi with new locking mechanisms.

Key words: mini-slings, urinary incontinence, mini-loops, synthetic loops 
D аже сегодня в век современного развития урологии недержание мочи при напряжении (НМПН) у женщин остается значительной социальной проблемой, которая крайне негативно влияет на качество их жизни $[1,2]$. Безусловно, недержание мочи не является угрожающим для жизни заболеванием, но часто может приводить пациенток к депрессиям и к затворническому образу жизни.

В течение последнего столетия различные петлевые операции используются при лечении недержания мочи при напряжении. Методы лечения недержания мочи неразрывно связаны с развитием урологии как науки. Доминирование концепции о сфинктерной недостаточности как основной причины недержания мочи привело к использованию различных мышечных петель, которые, как полагали многие авторы, могли нести функциональную нагрузку. Со временем различные петли стали применяться для создания дозированной инфравезикальной обструкции в области шейки мочевого пузыря. Последнее десятилетие было ознаменовано значительными успехами и достижениями в лечении недержания мочи у женщин. Синтез интегральной теории и создание современных синтетических слингов привели к настоящему прорыву в этой области и позволили избавить от недержания мочи большинство пациенток. Постоянно появляются новые модификации слинговых операций и разновидности синтетических материалов, из которых изготавливаются петли. Полученные сегодня данные дают право говорить, что, в целом, применение синтетических петель, устанавливаемых без натяжения в области средней уретры, позволяет избавить от недержания мочи значительное количество больных с этой медико-социальной проблемой во всем мире $[3,4,5]$.

\section{Развитие слинговых операций}

В начале 20 века Von Giordano описал первый уретральный слинг, который формировался из тонкой мышцы бедра (m.Gracilis) [6].

Последующие модификации этой методики предполагали использование лоскутов других мышц для формирования субуретральной петли, таких как m.Pyramidalis [7, 8, 9]. В основе применения слингов, формируемых из мышечных лоскутов, лежало предположение, что мышечная петля будет способна выполнять роль сфинктера мочевого пузыря. После внедрения слинговых операций в широкую практику, учеными того времени стали высказываться предложения о возможном применении других материалов для изготовления петли. В 1933 году Price [10] впервые описал фасциальный слинг (fascia lata). В основе современной позадилонной техники установки слинга лежит классическая работа, опубликованная Aldridge в 1942 году [11], в которой автор предлагал «трансплантацию» фасции 
прямой мышцы живота субуретрально - для «обеспечения поддержки уретры при повышении внутрибрюшного давления».

В 1962 году Narik и Palmrich на основании вышеописанной операции разработали собственную модификацию, которая заключалась в использовании лоскутов из апоневрозов наружных косых мышц живота. Апоневротические лоскуты формировались таким образом, что своей дистальной частью оставались прикрепленными к лону. Проксимальные концы лоскутов проводились позадилонно и фиксировались друг к другу под мочеиспускательным каналом [12].

В середине XX века среди ученых, занимающихся проблемами недержания мочи стала доминировать «теория трансмиссии давления», которую предложил Enhörling [13], он считал, что, вне зависимости от этиологии, недержание мочи развивается во время преодоления сопротивления уретры силами, изгоняющими мочу. Это создало основу для предложения и применения целого ряда операций, таких как Marshall-Marchetti-Krantz [14], направленных на смещение шейки мочевого пузыря.

В 1961 году Burch предложил выполнять позадилонную кольпосуспензию, при которой периуретральные ткани фиксировались к Куперовым связкам [15]. Эта операция на протяжении многих десятилетий считалась «зо- лотым стандартом» при лечении недержания мочи у женщин.

Основателем слинговых операций в Европе считается Steckel, а в США Ed.McGuire. Последним была предложена петлевая пластика с формированием лоскута из фасции прямой мышцы живота. Операция использовалась у больных с третьим типом недержания мочи, и, по мнению самого Ed.McGuire, являлась операцией выбора.

В 1978 году McGuire и Lytton дали «вторую жизнь» слинговым операциям, предложив применение изолированного фасциального лоскута, который устанавливался под уретру, в виде петли, комбинированным абдоминально-влагалищным доступом [16]. В том же году профессор Кан Д.В. использовал во время слинговой операции собственную кожу больной. Описывая различные оперативные пособия в своей монографии, Д.В. Кан указывал, что среди многочисленных операций при стрессовом недержании мочи, особенно, надежной считается петлевая пластика. Многие годы он разрабатывал эту методику. Профессор Кан Д.В. внес огромный вклад в развитие урогинекологии и реконструктивных операций у женщин. Им была предложена модифицированная операция ШтекеляКрантца с использованием кожного лоскута длиной до 20 см в комбинации с пластикой передней стенки влагалища. Подобные операции применялись у больных с третьим типом недержания 
мочи при напряжении, чаще всего это рецидивные формы. Это травматичное вмешательство сопровождалось значительной кровопотерей и длилось по началу несколько часов [17-22]. По-сути операции, предлагаемые авторами, были направлены на создание дополнительного обструктивного механизма в области шейки мочевого пузыря, так как основывались на предположении, что сфинктерная недостаточность лежит в основе недержания мочи у большинства больных. Однако при длительном послеоперационном наблюдении выяснилось, что эти методы лечения оказались малоэффективными [23].

\section{Синтетические петли}

Синтетические материалы применяются в лечении недержания мочи у женщин в течение многих лет. В 1962 году Williams и TeLinde [24] предложили технику операции с использованием синтетической петли для лечения недержания мочи, которая заключалась в проведении петли, изготовленной из полиэтилена тетрафталата, со стороны влагалищного разреза в позадилонное пространство через двухсторонние паховые разрезы. Далее петля фиксировалась к фасции прямой мышщы живота [25].

В 1970 году Morgan [26] описал методику установки синтетической петли комбинированным трансабдоминальным и трансвагинальным доступами с широкой мобилизацией шейки мочевого пузыря. Синтетическая петля изго- тавливалась из марлекса (монофиламентный полипропилен). Широкая петля проводилась под шейкой мочевого пузыря и фиксировалась к Куперовым связкам. В той же работе автор предложил применение синтетической петли без фиксации и натяжения, в своих последующих работах Morgan использовал короткую синтетическую петлю, располагавшуюся без всякой фиксации и натяжения под уретрой [27]. Эта работа предвосхитила последующие тенденции коррекции недержания мочи у женщин на несколько десятилетий.

Силиконовую петлю, прикрепляющуюся $к$ подвздошно-гребешковым связкам предложил Stanton в 1985 году [28], однако, этот хирургический доступ был оставлен в дальнейшем в связи с большим количеством послеоперационных осложнений [29].

В 1988 году Horbach [30] сообщил о другом способе коррекции недержания мочи. Описанный автором метод предполагал аппликацию периуретральной фасции над проксимальной уретрой, фиксацию средней части петли к периуретральной фасции, ее проведение к надлонному разрезу и прикрепление к фасции прямой мышцы живота. Тем же автором было предложено применение короткой синтетической петли, которая практически не отличается от современных [31].

Одним из распространенных методов использования свободной синтетической петли является операция DUPS 
(Distal Urethral Polypropylene Sling) описанная Rodriguez и Raz в 2001 году $[32,33]$. Этот метод был широко распространен в США и в меньшей степени в странах Европы. Авторы предложили использовать короткую полипропиленовую петлю, располагающуюся под средней уретрой. К латеральным краям петли прикрепляются лигатуры, которые проводятся ретроградно в позадилонную область при помощи специальных инструментов. Некоторые авторы предлагают фиксировать петлю к нижним ветвям лонной кости при помощи различных фиксирующих устройств. Следует отметить, что подобная техника приводит к значительному количеству осложнений и редко применяется сегодня [34].

В 1995 году шведский ученый Ульф Ульмстен (Ulf Ulmsten) предложил слинговую операцию с использованием синтетической петли, которая имела ряд принципиальных отличий от ранее применяемых методик [35]. В первую очередь, это касалось локализации петли, которая теперь располагалась под средней уретрой, а не под проксимальной, как было принято ранее. Кроме того, петля устанавливалась под мочеиспускательным каналом без натяжения, не создавая какой-либо обструкции. Эти новые принципы, сформированные Ульмстеном, наряду с использованием полипропилена для изготовления петли, оказали, несомненно, революционное значение на эффективность после- дующих операций при недержании мочи у женщин.

В 1997 году Amid [36] предложил классификацию синтетических материалов, которую используют и на сегодняшний день. Согласно этой классификации наиболее подходящими материалами, которые используют в урогинекологии, являются материалы, относящиеся к типу I. В целом же, идеальная синтетическая петля должна обладать несколькими качествами, а именно: биосовместимостью, инертностью, прочностью, макропористостью, быть доступной по цене. Монофиламентные макропористые полипропиленовые петли, представленные сегодня на рынке, более всего соответствуют таким требованиям.

Незадолго до этого, в 1990 году, Реtros и Ulmsten [37] предложили интегральную теорию недержания мочи. Интегральная теория предполагает, что стрессовое и императивное недержания мочи имеют общую этиологию, связанную с повреждением анатомических структур таза. Согласно современным представлениям о патогенезе недержания мочи, средняя уретра - это точка приложения множества факторов, участвующих в удержании мочи. В 1991 году Lose [38] доказал, что у женщин, страдающих недержанием мочи, давление в средней уретре значительно ниже, чем у женщин, не страдавших этим заболеванием. Для этого автор проводил измерения давления в проксимальной, 
средней и дистальной частях мочеиспускательного канала при помощи перманентного датчика.

Средняя часть уретры расположена в своеобразном миофасциальном гамаке, который фиксирует ее к костям таза, с помощью лонно-уретральной и уретротазовой связок. Как было сказано ранее, передняя стенка влагалища и входящая в ее состав пубоцервикальная фасция удерживают уретру снизу. Прижатие уретры к интактному миофасциальному гамаку, наряду со сфинктерным механизмом и замыкательными свойствами слизистой уретры, является фактором, необходимым для удержания мочи при резком повышении внутрибрюшного давления. Повреждение тканей передней стенки влагалища вызывает нарушение данного механизма. При декомпенсации остальных факторов удержания мочи, возможно, развитие недержания мочи при напряжении. Синтетическая петля, которая устанавливается без натяжения, не сдавливает уретру, а лишь создает жесткую и нерастяжимую опору под ее средней третью. Петля, расположенная таким образом, замещает поврежденный связочный аппарат уретры и пубоцервикальную фасцию. А при повышении внутрибрюшного давления уретра прижимается к нерастяжимой петле, что приводит к закрытию ее просвета.

Повреждение поддерживающих структур уретры и излишняя растяжимость передней стенки влагалища ослабляют вектор сил, направленных кпереди. Превалирование вектора, направленного кзади может приводить к открытию шейки мочевого пузыря. Растяжение рецепторов шейки - к ургентным позывам. Наличие синтетического протеза лонно-уретральной связки, которым является петля, препятствует преобладанию вектора, направленного кзади и удерживает мочевой пузырь в «закрытом» функциональном состоянии. Таким образом, можно предположить, что протезирование ослабленных связок уретры может устранить императивную симптоматику, связанную с открытием шейки мочевого пузыря.

\section{Позадилонные}

\section{и трансобтураторные слинги}

В настоящее время ни у кого не вызывает сомнения, что операции с использованием свободной синтетической петли являются основным методом устранения недержания мочи у женщин. Сегодня существует множество модификаций хирургических доступов для ее установки. Все эти методы можно разделить на две большие группы: позадилонные и трансобтураторные.

В 1995 году Ulmsten описал технику «амбулаторной процедуры под местной анестезией», при которой синтетическая петля проводится со стороны разреза во влагалище позадилонно. Эта техника операции получила название TVT (Tension-free Vaginal Tape). Сегодня накоплен огромный опыт выполне- 
ния этих операций во всем мире. Эффективность устранения недержания мочи согласно данным различных авторов колеблется от 67 до 96\% [39, 40]. Такое разнообразие в оценке лечения вызвано применением различных критериев успешности лечения. Но, несомненно, эффективность данного метода не только сравнима с предшествующими, но и превосходит их. Время оперативного вмешательства, госпитализации, реконвалесценции, а также необходимость применения обезболивающих препаратов в послеоперационном периоде значительно меньше при TVT, чем при других операциях [41].

Наиболее частым и серьезным осложнением позадилонного метода, отмеченным в литературе, остается повреждение мочевого пузыря. Имеются лишь единичные сообщения о невозможности диагностировать перфорацию мочевого пузыря в ходе операции, несмотря на выполнение цистоскопии [42].

Угрожающие жизни осложнения редко развиваются после применения синтетических петель. Согласно данным Manufacturer and User Facility Device Experience Database (MAUDE), являющимся подразделением FDA (Food \& Drugs Administration, США), с 1999 по 2008 год зарегистрировано девять летальных случаев, вызванных осложнениями TVT. Семь из них явились следствием интраоперационного повреждения кишечника, и в двух случаях были травмированы крупные сосуды.
Анализируя современную литературу, относящуюся к операции TVT, с 1996 по 2013 годы мы обнаружили более 10 рандомизированных клинических исследований по этой теме. Результатом трех из опубликованных работ с периодом послеоперационного наблюдения в 2 года являлось то, что операция TVT сравнима по эффективности с операцией Burch [43, 44, 45]. Ustin в 2003 [46] и Paraiso в 2004 годах [47] провели сравнение TVT с лапароскопической модификацией операции Burch и считают, что методы одинаково эффективны. Другие авторы сравнивали TVT в рандомизированных исследованиях с операциями ТОТ (deTayrac, 2004), SPARC [48], TVT-O [49], фасциальным слингом [50], дермальным слингом [51]. Во всех исследованиях операция TVT показала высокую эффективность и безопасность.

Несомненный интерес представляют данные, опубликованные Nilsson в 2004 году, которые относятся к группе пациентов, перенесших операцию в числе первых [51]. Автор сообщает о 80 из 90 прооперированных больных наблюдающихся более 7.5 лет, 81\% из них считают лечение успешным.

Трансобтураторный метод установки петли, примененный впервые Delorme [52] в госпитале Bishat (Франция) в 2001 году, еще более упростил оперативное пособие. Оригинальная техника предполагает проведение петли с помощью перфорирующего инструмента: 
с внутренней поверхности бедра к разрезу во влагалище через запирательные отверстия. Автор предполагал, что такой доступ минимизирует вероятность повреждения мочевого пузыря, и не потребует интраоперационной цистоскопии. Описанная автором операция получила известность как ТOT (Trans Obturator Tape). Delorme использовал синтетические петли Obtape, которые выполнены из нетканного полипропилена. В дальнейшем оказалось, что подобный материал часто приводит к эрозиям влагалища, и риск инфекционных осложнений при его использовании выше, чем при использовании плетеного материала с более крупными порами.

Бельгийский уролог DeLeval [53] опубликовал работу, в которой предлагал трансобтураторный доступ с проведением петли со стороны разреза во влагалище к кожному разрезу на бедре, то есть изнутри кнаружи. Операция получиланазвание TVT-O (Tension-free Vaginal Tape - Obturator). Трансобтураторный доступ установки свободной синтетической петли становится наиболее популярным. Сегодня по данным мировой статистики выполнено более 400000 операций.

Позадилонный метод установки свободной синтетической петли считается одним из самых эффективных и безопасных способов устранения недержания мочи у женщин. В то же время, операция TVT сопровождается различными осложнениями, такими как пер- форации мочевого пузыря (2,7-13,8\%), интраоперационные кровотечения (4\%), формирование гематом $(0,6-3,4 \%)$, задержки мочи (0,5-20\%), инфекции нижних мочевых путей (0,7-22\%) и вновь возникшая (de novo) гиперактивность мочевого пузыря (2,5-25\%) [54-56].

Сравнительный ретроспективный анализ позадилонного и трансобтураторного методов показал, что они одинаково эффективны [57]. При этом 89\% больных после TVT и 90\% после TVT-О не имели признаков недержания мочи через 1 год после операции.

Опубликованы несколько рандомизированных контролируемых исследований, сравнивающих TVT с TVT-O. Исследование, опубликованное Mansoor [58], включало 102 пациента, для которых метод оперативного вмешательства выбирался рандомизированно (48 TVT-O и 54 TVT). Результаты лечения недержания мочи были одинаковы в обеих группах и составили 93\% для TVT и 96\% для TVT-О соответственно. Императивные симптомы были устранены в $60 \%$ случаев после TVT и у $80 \%$ пациентов группы TVT-O. Обструктивное мочеиспускание, потребовавшее уретролиза, наблюдалось у пяти пациентов после TVT и одного после TVT-O. Итак, автор считает, TVT-O так же эффективен, как и TVT, но вызывает меньшее количество осложнений.

Другое рандомизированное сравнение позадилонного (TVT) и трансобтураторного (ТОТ) методов установки 
петли для лечения недержания мочи проведено DeTayrac [59]. Оказалось, что средняя продолжительность операционного времени практически в два раза меньше при трансобтураторном методе. Частота возникновения инфравезикальной обструкции не зависела от примененного метода. Сопоставимые данные получены и другими учеными, изучавшими эффективность позадилонного метода установки синтетической петли в лечении недержания мочи у женщин [60].

Рандомизированное клиническое исследование, опубликованное Liapis в 2006 году [61], подтвердило высокую эффективность методов при непродолжительном периоде послеоперационного наблюдения. По данным автора, продолжительность операции позадилонным доступом оказалась значительно выше, что связано с необходимостью выполнения цистоскопии.

Риск повреждения мочевого пузыря очень низок при использовании трансобтураторного доступа. Однако, такие осложнения были описаны Hermieu [62] и Krauth [63]. Последний считает, что выраженное цистоцеле увеличивает риск перфорации мочевого пузыря. Мinaglia [64] описывает 3 случая перфорации мочевого пузыря при TVT-O и считает необходимым выполнение интраоперационной цистоскопии.

В последнее время было предложено два новых доступа установки синтетической петли. Первый доступ представ- ляет собой «укороченный» вариант петли, устанавливаемой влагалищным доступом. Форма петли позволяет устанавливать ее как U-образно, наподобие позадилонногослинга, так и в форме гамака - трансобтураторный слинг. Техника операций не предполагает прохождение троакара трансобтураторно или позадилонно, что, по-видимому, должно минимизировать осложнения, связанные с этим.

Другой доступ, описанный Daher et al. в 2003 году, был назван предлонным (операция Prefyx PPS) [65]. Операция заключается в проведении петли со стороны передней стенки влагалища кпереди от лонного сочленения - предлонно. Теоретически такой доступ должен предотвратить возможность повреждения любых органов малого таза. Однако, на наш взгляд, риск повреждения сосудов и нервов клитора остается возможным.

Опыт применения операций с использованием стандартных субуретральных слинговых операций во всем мире превысил 10 лет. В отечественной литературе опубликовано много работ, которые заслуживают внимания [66, 67].

Отечественные авторы Касян Г.Р., Гвоздев М.Ю., Годунов Б.Н., Прокопович М.А, Пушкарь Д.Ю. в своем исследовании представили анализ опыта лечения недержания мочи у женщин с использованием субуретрального слинга TVT-O. Всего с 2001 по 2008 год была прооперирована 1081 пациентка. Из 
них 841 (77,8\%) страдала стрессовым недержанием мочи, 202 (18,62\%) смешанной формой недержания мочи с преобладанием стрессового компонента, а у 38 (3,52\%) имелось рецидивное недержание мочи. Первую группу составили пациентки со стрессовым недержанием мочи, прооперированные с использованием позадилонного доступа (операция TVT), во 2-ю группу вошли пациентки, прооперированные с применением субуретральнойетли , имплантированной трансобтураторным доступом (операция TVT-O). Третью группу составили пациентки со смешанной формой недержания мочи, которым была выполнена операция TVTO. Позадилонным доступом петля TVT была имплантирована 273 (25,25\%) пациенткам, трансобтураторным доступом TVT-O - 740 (68,45\%), другие субуретральные синтетические петли - 68 (6,3\%). Средний период наблюдения за больными составил 50,1; 31,1 и 32,6 месяцев в каждой из групп соответственно. Для объективной оценки эффективности авторы применяли кашлевую пробу, для субъективной- визуальную аналоговую шкалу. В группе TVT отрицательная кашлевая проба в послеоперационном периоде сохранялась у $85,58 \%$ пациенток, а в группе TVT-O у $84,36 \%$. Анализ частоты интраоперационных осложненийоказал, что вероятность возникновения последних не связана с возрастом, индексом массы тела и акушерским анамнезом пациен- ток. Отмечено, что перфорация мочевого пузыря и тазовая гематома чаще возникают при использовании позадилонного доступа. Трансобтураторный доступ сопряжен с более высоким риском травмы латеральных сводов влагалища, хотя не исключена и травма мочевого пузыря. Объективные и субъективные показатели эффективности лечения смешанной формы недержания мочи составили 86,15 и $87,69 \%$ соответственно. Авторы пришли к заключению, что операции с использованием субуретральных петель TVT и TVT-O являются безопасными и эффективными методиками лечения стрессового недержания мочи у женщин [68].

Многочисленными исследованиями было доказано, что синтетический слинг позволяет надежно устранить недержание мочи, однако, для женщин пожилого возраста с отягощенным соматическим статусом не всегда возможно выполнение оперативного лечения под общим наркозом. Использование трансобтураторных слингов зачастую приводит к диспареунии в послеоперационном периоде. Данные проблемы привели к разработке синтетических слингов других размеров. Технология их установки не предполагает выведение ленты на кожу и контакта со стенкой мочевого пузыря, что снижает вероятность интраоперационных осложнений и, в первую очередь, перфорацию мочевого пузыря. Оперативное лечение с использованием таких петель 
предполагает использование местной анестезии, что предоставило альтернативу пациенткам, не решавшимся ранее на объемные операции. Можно сказать, что с появлением минислингов и их ис- пользовании, в лечении недержания мочи появился новый этап.

публикация второй части статьив следующем номере журнала

\section{ЛИТЕРАТУРА}

1. Белова, А.Н. Шкалы, тесты и опросники в медицинской реабилитации / А.Н. Белова .- М.:Антидор. -2002. - 439 с.

2. Новик, А.А. Руководство по исследованию качества жизни в медицине / А.А. Новик. - СПб.: Олма. Пресс. 2002. $-314 \mathrm{c}$.

3. The prevalence of urinary incontinence in women in four European countries / S. Hunskaar, G. Lose, D. Sykes, S. Voss // BJU Int. -2004,Feb. Vol.93(3). - P.323-324.

4. Cardozo, L. Urinary incontinence in primary care / L. Cardozo, D. Staskin, M. Kirby // Oxford: is is Medical Medi. 2002. $-128 \mathrm{p}$.

5. Cardozo, L. A new questionnare to assess the quality of life of urinary incontinent women / L. Cardozo, C.J. Kelleher, V. Khullar // Br. J. Obstet. Gynecol. 1997. - Vol.104. - P.52-54.

6. Rodriguez, L.V. Polypropylene sling for the treatment of stress urinary incontinence / L.V. Rodriguez, S. Raz // Urology - 2001. - Vol.58. - P.783-785.

7. Goebell, R. Zur operativen Beseitgung der angeborenen IncontinentiaVersicae / R. Goebell // Dtschr Gynakol Urol 1910. - Vol.2. - P.187-191.
8. Frangenheim, P. Zur operativen behandlung der Inkontinenz der mannlichen Harnrohe / P. Frangenheim // Verh Dtsch Ges Chir - Vol.43. - P.149-156.

9. Stoeckel, E.P. Treatment of incontinence of urine in traumatic injuries of the sphincter muscle / E.P. Stoeckel // Zentralbl Gynakol - 1921. - V.45. - P.17-19.

10. Price P.B. Plastics operations for incontinence of urine and feces / P.B. Price // Arch Surg - 1933. - Vol.26. - P.10431048.

11. Aldridge, A.H. Transplantation of fascia for the relief of urinary incontinence / A.H. Aldridge // Am J Obstet Gynecol 1942. - Vol.44. - P.398-411.

12. Narik, G. A simplified sling operation suitable for routine use / G. Narik, A.H. Palmrich // Am J Obstet Gynecol 1962. - Vol.4. - P.400-405.

13. Enhörling, G. Simultaneous recording of intravesical and intraurethral pressure: a study on urethral closure in normal and stress incontinent women / G. Enhörling // Acta Chir Scand. - 1953. - Vol.32 P.285-307.

14. Marshall, F.V. The correction of stress incontinence by simple vesicourethral suspension / F.V. Marshall, A.A. Mar- 
chetti, K.E. Krantz // Surg Gynecol Obstet - 1949. - Vol.88. - P.509-518.

15. Burch, J.C. Urethrovaginal fixation to Cooper's ligament for correction of stress incontinence, cystocele, and prolapse / J.C. Burch // Am J Obstet Gynecol - 1961. - Vol.81. - P.281-290.

16. McGuire, E. J. Pubovaginal sling procedure for stress incontinence / E.J. McGuire, B. Lytton // J Urol. - 1978. - Vol.119 - P.82-84.

17. Кан, Д.В. Руководство по акушерской и гинекологической урологии. 2-е изд. перераб. и доп. / Д.В. Кан. - М.: Медицина. - 1986. - 488 с.

18. Кан, Д.В. Оперативное лечение недержания мочи у женщин / Д.В. Кан, К.А. Гапоян, В.Г. Петросян // Урология и нефрология. - 1971. - №4. - С.85-87.

19. Некоторые аспекты диагностики недержания мочи при напряжении у женщин / Д.В. Кан, Л.М. Гумин, Ю.М. Захматов и др. // Актуальные вопросы урологии. - Алма-Ата, 1981. -С.121-124.

20. Кан, Д.В. О модификации операции Лукича в терапии недержания мочи при напряжении у женщин / Д.В. Кан, , Г.Л. Егорова // Пробл. урол. и нерол. Кемерово, 1975. - Ч.1. - С.75-76.

21. Кан, Д.В. Диагностика и лечение недержания мочи при напряжении у женщин / Д.В. Кан, О.Б. Лоран, Б.В. Еремин // Методич. Разработки ММСИ им. Семашко. - М., 1987. - 55с.

22. Кан, Д.И. Руководство по акушерской и гинекологической урологии / Д.И. Кан. - 1978. - С.278-300.
23. Lobel, R.W. Long-term results of vaginal wall suburethral sling / R.W. Lobel, P.K. Sand // J Urol - 1997. - Sup.157. - Abstr.459.

24. Williams, T.J. The sling operation for urinary incontinence using Mersilene ribbon / T.J. Williams, R.W. TeLinde // Obstet Gynecol - 1962. - Vol.19. - P.241.

25. Nichols, D.H. The Mersilene mesh gauze hammock for severe urinary stress incontinence / D.H. Nichols // Obstet Gynecol - 1973. - Vol.41. - P.88-93.

26. Morgan, J.E. A sling operation using Marlex polypropylene mesh for treatment of recurrent stress incontinence / J.E. Morgan // Am J Obstet Gynecol 1970. - Vol.106. - P.369-377.

27. The polypropylene pubovaginal sling for the treatment of recurrent stress urinary incontinence / J.E. Morgan, D.M. Heritz, F.E. Stewart et al. // J Urol 1995. - Vol.154. - P.1013-1014.

28. Stanton, S.L. Silastic sling for urethral sphincter incompetence in women / S.L. Stanton, G.S. Brindley, G.S.Holmes // Br J Obstet Gynecol - 1985. - Vol.92. P.747-750.

29. Chin, Y.K., A follow-up of Silastic sling for genuine stress incontinence / Y.K. Chin, S.L. Stanton // Br J Obstet Gynecol - 1995. - Vol.102. - P.143-147.

30. Horbach, N.S. A suburethral sling procedure with PTFE for the treatment of genuine stress incontinence in patients with low urethral closure pressure / N.S. Horbach, J.S. Blanco, D.R. Ostergard // Obstet Gynecol - 1988. - Vol.71. - P.648-652. 
31. Staskin, D.R. The Gore-Tex sling procedure for female sphincteric incontinence / D.R. Staskin, J.M. Choe, D.S. Breslin // World J Urol - 1997. - Vol.15. P.295-299.

32. Rodriguez, L.V. Polypropylene sling for the treatment of stress urinary incontinence / L.V. Rodriguez, S. Raz // Urology - 2001. - Vol.58. - P.783-785.

33. Лоран, О.Б. Операция Раза в лечении недержания мочи при напряжении у женщин / О.Б. Лоран, Д.Ю. Пушкарь, В.В. Дьяков // Урол. и нефрол. 1996. - №1. - C.37-41.

34. Madjar, S. Pubic bone anchoring in the treatment of women with stress urinary incontinence: New applications to an old concept / S. Madjar, M. Beyar, O. Nativ // Int Urogynecol J Pelvic Floor Dysfunct 1998. - Vol.9. - P.416-418.

35. An ambulatory surgical procedure under local anesthesia for treatment of female urinary incontinence / U. Ulmsten, L. Herniksson, P. Johnson, G. Varhos // Int Urogynecol J Pelvic Floor Dysfunct 1996. - Vol.7. - P.81-85.

36. Amid, P.K. Classification of biomaterials and their related complications in abdominal wall hernia surgery / P.K. Amid // Hernia - 1997. - Vol.1. - P.2.

37. Petros, P.E. An integral theory of female urinary incontinence: experimental and clinical considerations / P.E. Petros, U.I. Ulmsten // Acta Obstet Gynecol Scand Suppl. - 1990. - Vol.153. - P.731.

38. Lose, G. Urethral pressure and power generation during coughing and volun- tary contraction of the pelvic floor in females with genuine stress incontinence / G. Lose // Br J Urol. - 1991. - Vol.67. P.580-585.

39. Wang, A.C. Tension-free vaginal tape. A minimally invasive solution to stress urinary incontinence in woman / A.C. Wang, T.S. Lo // J Reprod Med. 1998. - Vol.43. - P.429-434.

40. The tension-free transvaginal tape procedure in the treatment of female urinary stress incontinence: A French prospective multicentre study / M. Soulie, X. Cuvillier, A. Benaissa et al. // Eur Urol. - 2001. - Vol.39. - P.709-714.

41. Tension free vaginal tape versus Burch colposuspension for treatment of female stress urinary incontinence / E. ElBarky, A. El-Shazly, O.A. El-Wahab et al. // Int Urol Nephrol. - 2005. - Vol.37(2). P.277-281.

42. Buchsbaum, G.M. True occult bladder perforation during placement of tension-free vaginal tape / G.M. Buchsbaum, C. Moll, E.E. Duecy // Int Urogynecol J Pelvic Floor Dysfunct - 2004, Nov-Dec. Vol.15(6). - P.432-433.

43. Ward, K United Kingdom and Ireland Tension-free Vaginal Tape Trial Group. Prospective multicentrerandomised trial of tension-free vaginal tape and colposuspension as primary treatment for stress incontinence / K. Ward, P. Hilton // BMJ. - 2002,Jul. - Vol.13,Suppl.325(7355). P.67.

44. Liapis, A. Burch colposuspension and tension-free vaginal tape in the man- 
agement of stress urinary incontinence in women / A. Liapis, P. Bakas, G. Creatsas // Eur Urol. - 2002,Apr. - Vol.41(4). - P.469-473.

45. Ward, K.L. UK and Ireland TVT Trial Group. A prospective multicenter randomized trial of tension-free vaginal tape and colposuspension for primary urodynamic stress incontinence: two-year follow-up / K.L. Ward, P. Hilton // Am J Obstet Gynecol. - 2004, Feb. - Vol.190(2). P.324-331.

46. Tension-free vaginal tape compared with laparoscopic Burch urethropexy / Y. Ustun, Y. Engin-Ustun, M. Gungor, S. Tezcan // J Am Assoc Gynecol Laparosc. - 2003, Aug. - Vol.10(3). - P.386389. Erratum in: J Am Assoc Gynecol Laparosc. - 2003, Nov. - Vol.10(4). - P.581.

47. Paraiso, M.F. Laparoscopic Burch colposuspension versus tension-free vaginal tape: a randomized trial / M.F. Paraiso, M.D. Walters, M.M. Karram, M.D. Barber // Obstet Gynecol. - 2004, Dec. - Vol.104(6). - P.1249-1258.

48. TVT and Sparcsuburethral slings: a case-control series / H.P. Dietz, A.J. Foote, H.L. Mak, P.D. Wilson // Int Urogynecol J Pelvic Floor Dysfunct. - 2004, Mar-Apr. Vol.15(2). - P.129-31, discussion 131.

49. Retropubic compared with transobturator tape placement in treatment of urinary incontinence: a randomized controlled trial / E. Laurikainen, A. Valpas, A. Kivela et al. // Obstet Gynecol. - 2007, Jan. - Vol.109(1). - P.4-11.

50. Wadie, B.S. Autologous fascial sling vs polypropylene tape at short-term follo- wup: a prospective randomized study / B.S. Wadie, A. Edwan, A.M. Nabeeh // J Urol. - 2005, Sep. - Vol.174(3). - P.990-993.

51. Abdel-Fattah, M. Pelvicolpubovaginal sling versus tension-free vaginal tape for treatment of urodynamic stress incontinence: a prospective randomized threeyear follow-up study / M. Abdel-Fattah, J.W. Barrington, A.S. Arunkalaivanan // Eur Urol. - 2004, Nov. - Vol.46(5). P.629-635.

52. Nilsson, C.G. Seven-year follow-up of the tension-free vaginal tape procedure for treatment of urinary incontinence / C.G. Nilsson, C. Falconer, M. Rezapour // Obstet Gynecol. - 2004, Dec. - Vol.104(6). - P.1259-1262.

53. Delorme, E. Transobturator urethral suspension: mini-invasive procedure in the treatment of stress urinary incontinence in women / E. Delorme // Prog Urol. - 2001, Dec. - Vol.11(6). - P.1306-1313.

54. deLeval, J. Novel surgical technique for the treatment of female stress urinary incontinence: transobturator vaginal tape inside-out / J. deLeval // Eur Urol. - 2003, Dec. - Vol.44(6). - P.724-730.

55. Prospective analysis of complications of tension-free vaginal tape from The Netherlands Tension-free Vaginal Tape study / S.E. SchraffordtKoops, T.M. Bisseling, A.P.M. Heintz et al. // Am J Obstet Gynecol-2005. - Vol.193.-P.45-52.

56. Nilsson, C.G. The tension-free vaginal tape procedure is successful in the majority of women with indications for surgical treatment of urinary stress incon- 
tinence / C.G. Nilsson, N. Kuuva // BJOG - 2001. - Vol.108. P.414-419.

57. Autologous and synthetic urethral slings for female incontinence / K. Niknejad, L.S. Plzak, D.R. Staskin et al. // UrolClin North Am. - 2002. - Vol.29. P.597-611.

58. Comparison of retropubic and outside-in transobturator sling systems for the cure of female genuine stress urinary incontinence / A. Fischer, T. Fink, S. Zachmann, U. Eickenbusch // Eur Urol - 2005. - Vol.48. - P.799-804.

59. Mansoor, A. Surgery of female urinary incontinence using trans-obturator tape (TOT): a prospective randomized comparative study with TVT / A. Mansoor, N. Vedrine, C. Darcq // International Continence Society. - 2003. - Abstr.88.

60. A prospective randomized trial comparing tension-free vaginal tape and transobturatorsuburethral tape for surgical treatment of stress urinary incontinence / R. De Tayrac, X. Deffieux, S. Droupy et al. // Am J Obstet Gynecol - 2004, Mar. Vol.190(3). - P.602-608.

61. Outcomes and complications of transobturator tape (TOT): 1 year follow up / E. Kocjancic, R. Gherzi, S. Zaramella et al. // European Association of Urology. - 2004. - Abstr.309.

62. Tension-free vaginal tape versus tension-free vaginal tape obturator in women with stress urinary incontinence / A. Liapis, P. Bakas, M. Giner et al. // Gy- necol Obstet Invest. - 2006. - Vol.62. P.160-164.

63. Suburethral tape treatment of female urinary incontinence - morbidity assessment of the trans-obturator route and a new tape (I-STOP1): a multi-centre experiment involving 604 cases / J.S. Krauth, H. Rasoamiaramanana, H. Barletta et al. // Eur Urol. - 2005. - Vol.47. - P.102-106.

64. Bladder injury after TVT transobturator / J.F. Hermieu, A. Messas, V. Delmas et al. // Prog Urol. - 2003. - Vol.13. P.115-117.

65. Bladder injury during transobturator sling / S. Minaglia, B. Ozel, C. Klutke et al. // Urology. - 2004, Aug. - Vol.64(2). P.376-377.

66. Pre-pubic TVT: an alternative to classic TVT in selected patients with stress incontinence / N. Daher et al. // Eur J Obstet Gynecol Reprod Biol. - 2003. Vol.107. - P.205-207.

67. Лоран, О.Б. Хирургическое лечение рецидивных форм недержания мочи у женщин / О.Б. Лоран // Анналы хирургии. - 1996. - №3. - С.23-27.

68. Лоран, О.Б. Сравнительный анализ слинговой операции с использованием короткого кожного лоскута и операции TVT у больных с недержанием мочи / О.Б. Лоран, Д.Ю. Пушкарь // Тез.докл. II Российская научно-практическая конференция: «Недержание мочи у женщин: диагностика, лечение, результаты». - СПб, 2001. - С.41. 